\title{
Teaching Corner-"Lumps and Bumps": A practical guide to minor surgery for the Core Surgical Trainee
}

\author{
P. Rajan', A.Menon ${ }^{2}$, T.J. Crofts ${ }^{3}$ \\ 1. Beatson Institute for Cancer Research, Glasgow, G61 1BD \\ 2. Department of Surgery, Croydon University Hospital, Croydon, CR7 \\ $7 \mathrm{YE}$ \\ 3. Formerly Department of Surgery, Royal Infirmary of Edinburgh, \\ Edinburgh, EH16 4SA \\ Correspondence to: Dr. Prabhakar Rajan \\ E-mail:p.rajan@beatson.gla.ac.uk;
}

\begin{abstract}
The first local anaesthetic operating list faced by a Core Surgical Trainee (CT) can appear a daunting task. Fresh from Foundation Year (FY) posts, (s)he will lack experience in basic surgical techniques. At present, there is no formal training in minor surgical skills for FY doctors, and exposure to operative surgery can be variable. This review provides an introduction and practical guide to the operative management of minor surgical pathologies.

\section{Introduction}

The first local anaesthetic operating list faced by a Core Surgical Trainee (CT) can appear a daunting task. Fresh from Foundation Year (FY) posts, (s)he will lack experience in basic surgical techniques. Ascertaining the diagnosis and confidence in surgical technique are the keys to success and are only possible through adequate guidance and supervision. At present, there is no formal training in minor surgical skills for FY doctors, and exposure to operative surgery can be variable. This review is a practical guide to the operative management of some minor surgical pathologies. We hope to provide some guidance to the junior core surgical trainee to ensure that procedures are performed safely.
\end{abstract}

\section{General principles of minor surgery - "What to cut, where to cut, when to cut"}

Patients are often listed directly onto a local anaesthetic list and may not necessarily have been seen in clinic beforehand. It is always the responsibility of the operating surgeon to decide whether the procedure can be performed under local anaesthesia or warrants sub-speciality referral. Most of the lesions encountered are dermatological in origin and can be easily dealt with under local anaesthesia, but there are some caveats.

Suitable lesions for excision include sebaceous cysts, lipomas and intra-dermal pigmented naevi. Those to be avoided can be separated into 4 categories:

1. Type: Certain pigmented lesions are potentially malignant and should be avoided, as should lesions likely to be basal cell and squamous cell carcinomas.

These often occur on the face and it is often difficult to produce good aesthetic results operating around the eyes or nose. Referral to Plastic Surgery is warranted to obtain the best cosmetic results.

2. Site: Sound knowledge of surgical anatomy is of particular importance whilst operating in neck and other areas. The accessory nerve is prone to damage in the posterior triangle as is the lateral popliteal nerve around the knee. In the mandibular areas, a small discharging lesion may represent an underlying dental sinus. Breast lesions have a higher incidence of malignancy and can be difficult to anaesthetize, so should be excised under a general anaesthesia by a Breast Surgeon. Axillary lumps can frequently be deceptively deep seated, and difficult to manage under local anaesthesia.

3. Size: Larger lesions may be difficult to anaesthetise, and have a larger blood supply and can pose a problem in the absence of diathermy.

4. Depth: Ambitious excision margins can be difficult to close with good aesthetic results.

Wound infections are one of the commoner complications of surgery and small wounds are not exempt from this particular problem. Patient-related risk factors include general health and co-morbidities; in particular, diabetes and oral corticosteroids can delay wound healing. Surgeons should avoid operating on infected sites; for example, an infected sebaceous cyst should be incised and drained, or treated with antibiotics prior to formal excision. Operating on fully anticoagulated patients or patients on antiplatelet agents should be avoided as intra-operative bleeding can pose a problem in the absence of diathermy. These patients should stop their anticoagulation or antiplatelet agent(s) prior to surgery, or be admitted for heparinisation where indicated.

\section{Anaesthesia}

Safe and effective local and regional anaesthesia remains is a vital component of minor surgery and therefore a sound knowledge of anaesthetic agents (Table 1 ) and techniques is required. A fast acting agent such as lidocaine (0.5-2\%) can be used in most cases. A standard rule is that the smallest possible needle should be used to administer the least possible amount of local anaesthetic agent. Infiltration should be as wide as possible around the lesion without entering the lesion itself and preferably through an already anaesthetised area of skin. Infiltration should take place before scrubbing up to allow time for the agent to work. Long and short acting agents can be mixed in the initial infiltration (e.g. lidocaine and bupivicaine) to provide a rapid yet long-lasting effect.

Epinephrine mixtures (e.g. lidocaine with epinephrine) can be used to achieve a bloodless field and improve anaesthesia; here, the degree of blanching indicates the extent of anaesthesia. Epinephrine should never be used in the extremities such as the toes or nose. Ethylchloride spray can provide adequate anaesthesia to perform a rapid procedure, such as the incision and drainage of an abscess.

A digital nerve (ring) block is most commonly used for anaesthesia of digits.

After skin preparation, a tourniquet is applied to the digit using the finger portion of a latex glove without the tip or a section of Foley catheter secured with an artery forceps.

Using an orange $25 \mathrm{G}$ needle, the anaesthetic agent (e.g. 1\% lidocaine) should initially be infiltrated into the dorsum of the digit over the extensor tendon. Epinephrine should never be used, as inadvertent administration into the digital artery will cause ischaemia.

The needle should be advanced through the anaesthetized area to the lateral and medial borders of the phalanx where the digital nerves lie. Two to three $\mathrm{mL}$ of anaesthetic agent should be administered, which will usually take about 10 minutes for full effect. 
Toxicity and anaphylaxis to local anaesthetic agents can occur due to overdosage or inadvertent intravenous administration. Clinical signs and symptoms of toxicity include peri-oral tingling, paraesthesia, drowsiness, seizures, paralysis, coma and cardiovascular collapse. In this scenario immediate resuscitation is necessary.

\section{Incisions, Closure and Scars}

In general, incisions should be made vertically following Langer's lines along skin increases. Exceptions include hairy scalp area, where one should consider making incisions in the direction of which hair is combed. In females, on the back, one should be careful not to generate scars that may snag or catch on bra straps. When making the incision, a sharp 15-blade will suffice for smaller excisions and biopsies, but larger lipomas and toenail surgery may require a 10-blade.

Good closure is important in order to achieve acceptable cosmesis and prevent risk of wound infection or breakdown. Selection of suture material is important. For skin closure, a non-absorbable monofilament such as prolene or ethilon can be used. For subcuticular closure braided (e.g. vicryl or dexon) or monofilament sutures can be used.

Steristrips are often applied over the wound, and in some cases skin edges can be adequately opposed using steristrips alone.

In order to provide the best cosmetic result, the finest diameter of suture material possible should be used for as little time as possible. 6/0 sutures are recommended for the face, and 4/0 for elsewhere. For wounds on the face, sutures should be left in-situ for 5 days, on the scalp or torso, 8 to 10 days will be required respectively. Subcuticular suturing and steristrips are often used as they appear to provide the best cosmetic result. Monofilaments are preferable to braided sutures are the latter harbour bacteria in the filaments and are therefore a greater infection risk.

Good knot tying technique is essential; knots should be applied on the same side and at 90 degrees to the wound. Good tension-free approximation of skin edges, with edge evertion is important. Plain interrupted suturing or subcuticular suturing can be used if the remaining cavity is not deep. Mattress sutures can be used to obliterate any dead space.

Patient and site selection can be important in ensuring a good cosmetic result; some ethnic groups and areas are prone to scarring. Afro-Caribbean individuals are prone to keloid scars, and clues can be gained from previous scars. The area from the nipple to chin sometimes scars badly and care must be taken when operating in this area.

\section{Excision of sebaceous cyst}

A sebaceous or epidermoid cyst is an intradermal lesion caused by a blocked sebaceous duct. The cyst enlarges due to continued production of sebum and characteristically contains caeseous material. The key to differentiating this lesion from a dermoid cyst lies in identifying the puntum. Common sites for occurrence include the back, scalp and ears. Differential diagnosis in the face and neck include parotid tumours, branchial cysts or dermoid cysts.

The indications for removal of sebaceous cysts are threefold: Pain, cosmesis, and infection. The skin overlying the cyst and around it is infiltrated with local anaesthetic, taking care not to puncture the cyst wall. Incisions are variable and can be made vertically through the punctum, around the punctum or by excising an overlying ellipse of skin. The incision is continued to the superior surface of the cyst capsule and the plane between the capsule and surrounding tissue dissected out using fine scissors, artery forceps or a scalpel. If the wall of the cyst is punctured, care must be taken to ensure that all components of the wall are excised. Recurrence is prevented by excising both the cyst and cyst wall en-bloc. If the remaining cavity is large, a mattress suture may be necessary to obliterate the dead space. If there is any suspicion of the diagnosis the cyst should be sent for histological examination.

\section{Excision of lipoma}

A lipoma is a benign fatty tumour that usually occurs superficially in the subcutaneous tissue. Other sites include deeper planes with attachment to fascial layers and in the retroperitoneum. Superficial lipomas can occur anywhere on the body but are often found on contact areas over extensor surfaces. Lipomas can grow to a considerable size; they are usually encapsulated and lobulated with fibrous attachments. Multiple lipomata can occur in rare familial conditions such as Dercum's disease although malignant change is rare. Differential diagnoses include fibroadenomas, neurofibromas, sebaceous cysts and vascular lesions.

The indications for surgical excision are fourfold; size, pressure effects, pain and cosmesis. Larger lipomas are not suitable excision under local anaesthesia as there may be deeper fascial involvement. The face and hands are areas where there is a risk of damage to underlying structures such as the facial nerve and tendons, and specialist referral is warranted.

The anaesthetic agent should be infiltrated into the skin and deeper tissues. A vertical incision is made, along Langer's lines, over the lesion and down onto the capsule. The tissue planes are identified by blunt dissection and the lipoma is teased out. In some cases, very little dissection is required and applying pressure to the surrounding skin can squeeze the lesion out. If the lipoma is very large, there may be a significant remaining cavity and care should be taken to obliterate this dead space in the closure.

\section{Excision of skin lesion}

There are many skin lesions encountered in surgical practice that are amenable to excision under local anaesthesia. Skin tags, papillomas, seborrhaeic warts, pigmented naevi, histiocytomas and epitheliomas can all be excised. Many can be diathermized or excised under local anaesthesia. Other means of biopsy include incision biopsy of a rash or lesion to ascertain a diagnosis and punch biopsy. Skin tags are often multiple and therefore have to be approached under general anaesthesia. Malignant-looking facial lesions (e.g. keratoacanthoma) should be referred to a Dermatological specialist.

Indications for removal are threefold; cosmesis, pain and diagnostic. Where the diagnosis is in doubt, prior referral to a Dermatologist is necessary. The prognosis from malignant melanoma depends on excision margins. Lesions should be anaesthetized in a wide field and elliptical incisions made in the direction of Langer's lines. All lesions should be sent for histological examination. If a large defect is left, deep closure with an absorbable suture is advisable.

Only lesions less that $1 \mathrm{~cm}$ in diameter and away from the face are suitable for excision biopsy. Incisions should be made down to fatty tissue include the full thickness of dermis with a margin of normal skin around the lesion. Pathology 
specimens should be orientated with a stitch to facilitate reexcision if the margins are involved as part of a malignant process.

\section{Toenail procedures: Wedge excision and Zadek's procedure}

An ingrowing toenail is caused by the edge of the nail penetrating into the adjacent soft tissue causing pain, inflammation and infection. It commonly affects the big toe in young

adults and is due to incorrect footwear and poor nail care. Indications for surgical intervention are twofold; pain and infection, although pre-operative treatment with antibiotics is not usually required. Initial treatment is either by simple avulsion of the nail, or by wedge excision of the side portions. However, even with satisfactory nail care, recurrence is high. Therefore surgical excision (Zadek's Procedure ${ }^{3}$ or chemical ablation of the nail bed may be warranted for effective treatment.

The digit is prepared in the usual manner and anaesthesia is achieved with a ring block. In a wedge excision, a section of nail is excised from the affected side, by incision through the nail and the nailbed into the proximal germinal matrix at the angle of the nail. Using a fine tissue forceps, a wedge of nail is lifted from the toe and underlying portion of nailbed dissected out and curetted to prevent recurrence.

The angular portion of the wound at the nail edge is secured with a non-absorbable suture and a nonadherent dressing is applied. The tourniquet is removed and the toe is bandaged with a firm dressing or tubi-grip. Post-operatively, the foot should be elevated for 24 hours, and weight bearing allowed as pain permits. Sutures and dressing should be removed after 12 to 14 days.

Chemical ablation of the nail-bed with phenol (phenolisation) is a less destructive, less painful process and causes less bleeding. Following avulsion of the nail, angular incisions should be made at the nail bed corners, to allow access for curettage, and application of phenol. The lattercan be achieved with the use of cottonbuds or eye swabs. Phenolisation should be maintained for around 3 minutes, and followed by vigorous ethanol and saline washout. A recent Cochrane review suggested that simple nail avulsion combined with the use of phenol is more effective at preventing symptomatic recurrence, as compared with surgical excision (Rounding, 2003). This is, however, at the cost of increased risk of postoperative infection.

A Zadek's procedure is usually reserved for severe recurrent cases or onychogryphosis. Usually, a bilateral wedge excision has been attempted.

Complications of a Zadek's procedure and other toenail surgery include sepsis, osteomyelitis of the distal phalanx, ischaemia due pressure bandages and recurrence due to poor technique. Under ring block, an incision is made along the nail margin and at each angle of the nail extending proximally to the transverse skin crease just distal to the interphalangeal joint. The flap is elevated, the nail excised using artery forceps or a Macdonald's elevator, and germinal matrix dissected away. The matrix should be excised proximally to the origin of the extensor tendon. Angular sutures are applied and the wound is dressed as per a wedge excision. If the toe is infected, the nail can be removed and a delayed Zadek's procedure can be performed at 2 months.

\section{Conclusion}

We hope that the above review will provide the Core Surgical Trainee doctor an introduction to the principles of minor surgery. There is, however, no substitute for hands-on experience, adequate supervision and training for development of basic surgical skills. Undertaking minor surgery is an excellent foundation upon which further operative skills can be built with repeated exposure and practice.

\section{References}

1.Andrews, S. MRCS Core Modules: Essential Revision Notes 2nd Edition. Pastest 2002 Cracknell, I.D., Mead M.G. Atlas of Minor Surgery. Churchill Livingston 1997

2.Kirk, R.M., Winslet, M. Essential General Surgical Operations 1st Edition. Churchill Livingston 2001

3.Zadek, F.R. Obliteration of the nail bed of the great toe without shortening the terminal phalanx. Journal of Bone and Joint Surgery 1950. 32B: 66-67

4.Rounding, C; Bloomfield, S Surgical treatments for ingrowing toenails. Cochrane Database of Systematic Reviews. 1, 2003

\section{Acknowledgements}

We are grateful to $\mathrm{Mr}$ Michael Lim and patients from Wharfdale Hospital, Otley, UK for assistance with images. We thank Mr Imran Ahmad (Beatson Institute) for proofreading of the manuscript and suggestions. 